\title{
WIND, GEOTAIL, and GOES 9 observations of magnetic field dipolarization and bursty bulk flows in the near-tail
}

\author{
J.A. Slavin', D.H. Fairfield', R.P. Lepping ${ }^{1}$, A. Szabo', M.J. Reiner', M. Kaiser', \\ C.J. Owen ${ }^{2}$, T. Phan ${ }^{3}$, R. Lin ${ }^{3}$, S. Kokubun', T. Mukai', T. Yamamoto ${ }^{5}$, H.J. Singer ${ }^{6}$, \\ S.A. Romanov ${ }^{7}$, J. Buechner ${ }^{8}$, T. Iyemori ${ }^{9}$, and G. Rostoker ${ }^{10}$
}

\begin{abstract}
The WIND, GEOTAIL, and GOES 9 spacecraft were all in the nightside magnetosphere and Interball was in the solar wind when a substorm began at $\sim 07: 25$ UT on April 18, 1996. WIND and GEOTAIL were located at downstream distances of $\mathrm{X}$ $=-12$ to $-14 R_{\mathrm{c}}$. The separation between these spacecraft was $\sim 10$ $\mathbf{R}_{\mathrm{c}}$ in the $\mathrm{Y}$ direction and less than $\sim 1 \mathbf{R}_{\mathrm{e}}$ in $\mathrm{Z}$. While the northward field component at GOES 9 began to increase immediately, little or no dipolarization was observed at WIND and GEOTAIL until a series of very rapid $\mathbf{B}_{2}$ increases were observed 25-30 min later. About 1-2 min prior to each dipolarization event at WIND and GEOTAIL an earthward flow burst with peak speeds of $100-500 \mathrm{~km} / \mathrm{s}$ was initiated. The duration of these bursty bulk flows were 1-7 min. These unique observations are interpreted as strong evidence for the existence of spatially localized, but sometimes temporally overlapping flow bursts in the near-tail during substorm expansion phase which result, cumulatively, in sufficient earthward magnetic flux transport to produce the well known dipolarization at geosynchronous orbit.
\end{abstract}

\section{Introduction}

The most basic prediction of Dungey's (1961) theory of magnetospheric convection is that events consuming large amounts of energy, like substorms, must be accompanied by tailward flows and southward $B_{z}$ in the distant plasma sheet and earthward flows and northward $B_{z}$ in the near plasma sheet as lobe magnetic energy is dissipated in the acceleration of charged particles and the driving of electrical currents. GEOTAIL and ISEE 3 have provided direct confirmation of Dungey's fast anti-

\footnotetext{
'NASA/GSFC, Greenbelt, MD, USA

${ }^{2}$ Astronomy Unit, Queen Mary and Westfield College, London, U.K.

${ }^{3}$ Space Sciences Laboratory, University of California, Berkeley, CA, USA

${ }^{4}$ Solar-Terrestrial Environment Laboratory, Toyokawa, Japan

${ }^{5}$ Institute for Space and Astronautical Sciences, Tokyo, Japan

${ }^{6}$ NOAA Space Environment Center, Boulder, CO, USA

${ }^{7}$ Space Research Inst., Russian Academy of Sciences, Moscow, Russia

${ }^{8}$ Max-Planck-Institut fuer Aeronomie, Katlenburg-Lindau, Germany

${ }^{9}$ Data Analysis Center for Geomagnetism and Space Magnetism, Kyoto Univ., Kyoto, Japan

${ }^{10}$ Dept. of Physics, University of Alberta, Edmonton, Canada

Copyright 1997 by the American Geophysical Union.

Paper number 97GL00542.

0094-8534/97/97GL-00542\$05.00
}

sunward flows and southward magnetic fields in the more distant tail. The inner tail, however, has proven to be much more complex. Direct plasma sheet measurements are difficult to obtain because the plasma sheet thins dramatically just prior to substorm onset (see review by Fairfield, 1988). Observations of high speed sunward plasma sheet flows earthward of $X \sim-20 R_{e}$, when available, last for only a few min (Baumjohann et al., 1990). Angelopoulos et al. (1992) has termed these short duration, fast earthward flows bursty bulk flows (BBFs) and shown that they appear to carry enough northward $B_{z}$ to complete Dungey's magnetospheric flux transfer cycle. Finally, there is often a lack of clear one-to-one correlations between substorm onsets and BBFs in the near tail (Lopez et al., 1994). This has led to the suggestion by Angelopoulos et al. (1996) that reconnection and BBFs may be limited to certain local time sectors or "channels". Hence, the probability of a spacecraft being situated to observe BBFs at the onset of a given substorm could be quite remote.

An important aspect of the changes in magnetic field topology and the re-distribution of electrical currents within the magnetosphere during substorms is the formation of the substorm current wedge (SCW) $\Delta$ significant frartinn of the crocs-tail current in the near-tail is diverted via field-aligned currents (FACs) down into the eastern side of the midnight sector of the auroral oval at substorm onset, where it adds to the westward electrojet and then returns to the tail via outward FACs on the western side. Within the SCW the magnetic field relaxes toward a more dipolar configuration marked primarily by an enhanced northward magnetic field component. The manner in which the SCW initially forms and spreads longitudinally (Kokubun and McPherron, 1981) and radially down the tail (Jacquey et al., 1991) is still the subject of active research and conflicting theoretical models (e.g., Hesse and Birn, 1991; Lui, 1996; Rostoker, 1996).

\section{WIND - Geotail - GOES 9 Substorm Observations}

The trajectories of WIND and GEOTAIL on April 18, 1996 are displayed in Figure 1 along with average bow shock and magnetopause boundaries. These spacecraft were located in the near-tail at downstream distances of $X=-12$ to $-14 R_{e}$. This constellation is unique as compared with previous studies by virtue of the wide separation in $Y$ of the most distant spacecraft. Unfortunately, no energetic particle observations from the Los Alamos National Laboratory instruments in geosynchronous orbit or auroral images from POLAR are available for this substorm.

A summary of the Interball $B_{z}$ (panel $A$ ), CANOPUS (panel B), GOES 9 and Pi2 events (panel D), WIND (panels C, E-H) and GEOTAIL (panels I-L) observations taken between 07:00 and 10:00 UT are presented in Figure 2. The onset of the substorm at $\sim 07: 25$ UT follows an interval of IMF $B_{Z}<0$ at Interball and is marked by 1 ) a decrease in the CANOPUS CL index (which pro- 


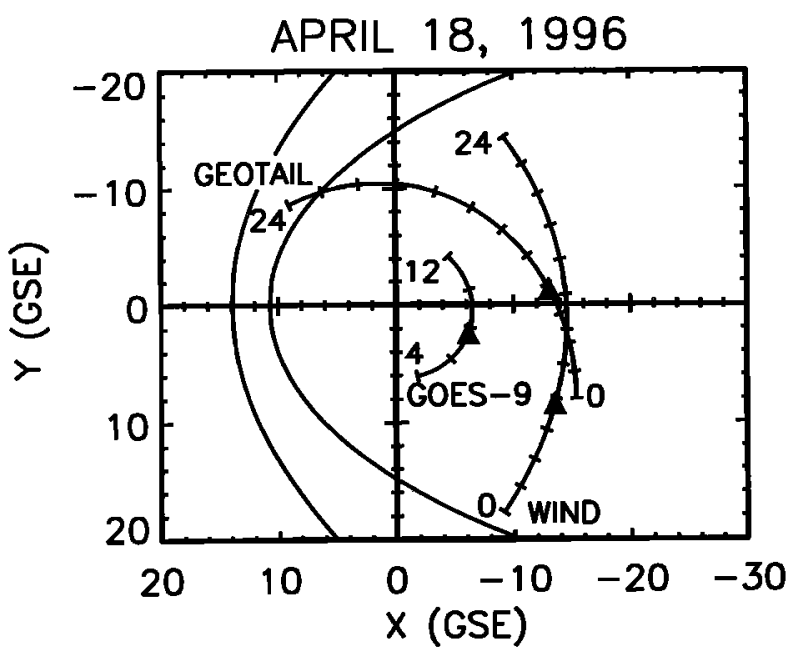

Figure 1. Spacecraft trajectories on April 18, 1996 have been projected into the GSE X-Y plane and plotted relative to average bow shock and magnetopause surfaces. The locations of these spacecraft at substorm onset are indicated with triangles.

vides a relative measure of westward electrojet intensity in the Canadian sector), 2) a strong increase in auroral kilometric radiation remotely measured at WIND, 3) the initiation of dipolarization in the GOES 9 field measurements and 4) the occurrence of multiple Pi2s at Mineyama in Japan and CANOPUS (marked with closed and open arrows, respectively, in panel D).

Examination of the total field strength and ion plasma $\beta_{i}$ displayed in Figure 2 (dotted line, panel $\mathbf{H}$ ) indicates that WIND was in the north lobe at the time of substorm onset. Over the next $\sim 30$ min the total field intensity in the lobe first increases and then slowly decreases to a level about $20 \%$ below its peak. It was not until several min before the first dipolarization seen by WIND at 07:59 UT that plasma sheet expansion placed this spacecraft in the central plasma sheet. GEOTAIL, in contrast, was in the plasma sheet or PSBL (i.e., $\beta_{i}>1$ ) for almost the entire interval except for 07:49 to 07:55 UT when plasma sheet thinning placed it briefly in the north lobe. After 09:30 UT both spacecraft gradually exited the plasma sheet into the south lobe.

Dipolarization is first observed at GOES 9 (LT 22:35; panel D) immediately following onset at 07:25 UT. While WIND was in the north lobe, it observed a slow increase in $B_{z}$ between 07:35 and 07:56 UT (panel G) simultaneous with a steeper increase in the northward field component at GOES 9. The plasma sheet thickening and engulfing of WIND at $\sim 07: 57$ UT is followed by a series of sharp pulses of $+B_{z}$ or "dipolarizations" beginning at 07:59 UT (marked with horizontal underlines in panel G). GEOTAIL also observed little or no dipolarization until a series of dipolarization events were observed beginning at 07:52 UT or about $7 \mathrm{~min}$ before similar events were seen at WIND. Except for the initial dipolarization, where the spacecraft was transitioning from the lobe to PSBL, these events all took place with GEOTAIL in the plasma sheet. Finally, it is interesting to note that some of the Pi2s (marked with arrows in panel D) and AKR intensifications are closely correlated with flow bursts at WIND and GEOTAIL while others are not.

The magnetic fields at WIND show that just before the first dipolarization event this spacecraft found itself immersed in not just the central plasma sheet, but in the cross-tail current layer as evidenced by the dominance of the $B_{z}$ field and the high $\beta$, values. GEOTAIL, in contrast, was in the north lobe at the time of the first steep dipolarization. In each case the dipolarization events at these spacecraft were accompanied by 1-7 min bursts of earthward flow (underlined in panels $\mathrm{E}$ and $\mathrm{I}$ ). Based upon the location of GEOTAIL (see Figure 1) just dawnward of midnight and WIND in the middle of the dusk half of the tail, it appears that channels of dipolarizations and earthward flow bursts may have been occurring across a region of at least $10 R_{e}$ width in the $Y$ direction. Alternatively, a single channel of dipolarization with BBFs at its edges may have expanded eastward and westward (e.g., see Figure 17 of Angelopoulos et al., 1996) to intercept these spacecraft.

Assuming that the integrated widths of the BBFs have a dawndusk extent of $10 R_{c}$, we have calculated the rate of magnetic flux transport earthward. In agreement with Angelopoulos et al. (1992; 1996), we find that these rates of flux transfer, several times $10^{5}$ $\mathrm{Wb} / \mathrm{s}$ (or $10^{4} \mathrm{~Wb} / \mathrm{R}_{\mathrm{c}} / \mathrm{s}$ ), and the integrated flux transport, $\sim 10^{8} \mathrm{~Wb}$ (or $10^{7} \mathrm{~Wb} / \mathrm{R}_{\mathrm{e}}$ ), are consistent with these events constituting the final return leg of Dungey's reconnection driven magnetic flux transfer cycle.

Later in the substorm recovery phase, Figure 2 shows additional flow burst events which were observed at WIND, but not seen by GEOTAIL despite its being located much closer to midnight. This difference between the two spacecraft may be associated either with WIND's still being located near the center of the cross-tail current layer or, again, due to the restriction of the fast earthward flows to narrow channels. Examples of these very brief flow bursts during the recovery phase are the events starting at $\sim$ 08:35 and $\sim$ 08:43 UT. Both events were associated with $\mathrm{Pi} 2 \mathrm{~s}$ and the initiation of brief $\mathrm{AKR}$ enhancements.

\section{Dipolarization and Flow Bursts}

An expanded view of the expansion phase dipolarization events and flow bursts is presented in Figure 3. The three panels present the north-south magnetic field component at highest time resolution taken by GOES 9 (2/s), GEOTAIL (16/s) and WIND (22/s). In addition, the $V_{x}$ component of the bulk flow speed at WIND and GEOTAIL is shown (dotted line) and the times of Pi2s observed by the CANOPUS chain of magnetometers and at Mineyama are indicated by vertical dashed lines. The interval begins with $\mathrm{B}_{\mathrm{z}} \sim 0$ at both GEOTAIL and WIND and a typical pre-substorm $\sim 50 \mathrm{nT}$ northward field at GOES 9 . Nearly coincident with the substorm onset and the first Pi2, a brief 1-2 min pulse of southward magnetic field with similar amplitudes of 5-7 nT was observed at all 3 spacecraft. The similarity of the amplitude of this perturbation at all 3 spacecraft implies that the current source must be distant and/or very large scale relative to the $\sim 10 \mathbf{R}_{\mathbf{e}}$ spacing of WIND, GEOTAIL and GOES 9. These southward tiltings of the field at substorm onset have been reported previously in AMPTE/CCE magnetic field dipolarization events at $X \geq-8.8 R_{c}$. Ohtani et al. (1992) attributed such events to a short duration "explosive growth phase" during which the intensity of the cross-tail current layer tailward of CCE increased suddenly just before substorm onset.

Another Pi2 is observed at 07:35 UT just after a weak 100 $\mathrm{km} / \mathrm{s}$ flow burst and a small pulse of northward $B_{\mathrm{z}}$ at GEOTAIL and just before a similar event at WIND. However, between substorm onset and the initial dipolarization at GEOTAIL at 07:52 UT, there are no fast flows or $\pm B_{z}$ variations capable of transporting significant amounts of magnetic flux. Since GEOTAIL was in the plasma sheet not far from midnight for most of this interval, the most likely reason for not observing such flows should not have been the $Z$ position of the spacecraft, but rather the localiza- 


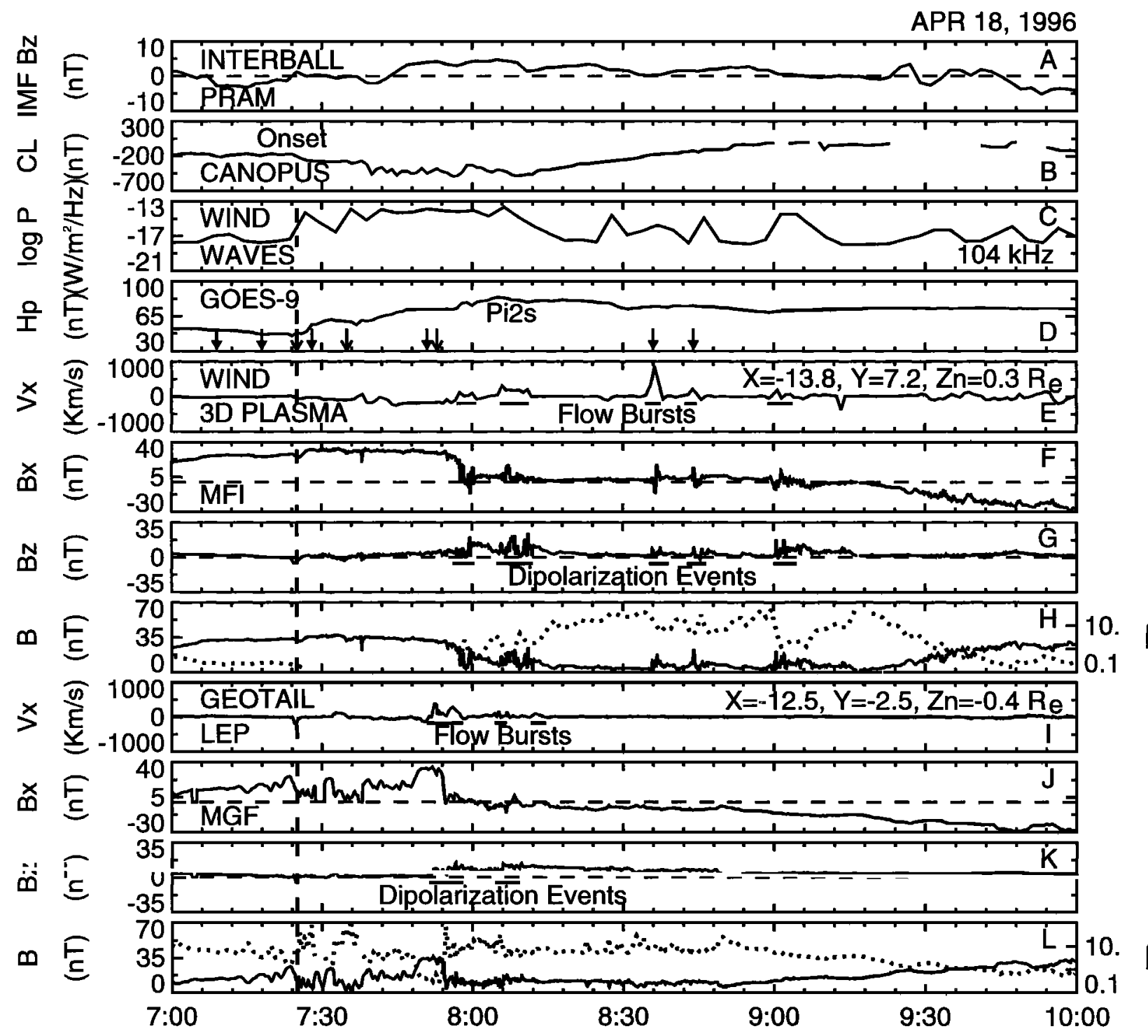

Figure 2. WIND, GEOTAIL, GOES 9, Interball, and CANOPUS measurements are displayed for the 07:25 UT substorm. A dashed vertical line marks the onset time determined from the westward electrojet, AKR power, and initial dipolarization signatures at the three spacecraft. The spacecraft positions in GSM $X$ and $Y$ and distance from the neutral sheet $\mathrm{Zn}$ (Fairfield, 1980) are given in panels $\mathrm{E}$ and $\mathrm{I}$.

tion of earthward flows and their bursty nature in $\mathrm{Y}$ as argued by Angelopoulos et al. (1996). Pi2 events were later seen at 07:51 and 07:53 UT. These Pi2s were observed just before the minimum in $\mathrm{CL}$, i.e., the peak of the expansion phase, and straddle the first strong dipolarization/flow burst event at GEOTAIL at $X=-12.5 R_{c}$ near midnight. A similar event is seen by WIND at $X=-13.8 R_{e}$ in the middle of the dusk half of the tail $8 \mathrm{~min}$ after the initial flow burst at GEOTAIL. A second set of dipolarizations and BBFs are observed at both WIND and GEOTAIL 6-12 min later and with significant temporal overlap between the events seen at the two spacecraft. In each case the sudden increase in $\mathrm{B}_{\mathrm{Z}}$ is preceded by -1-2 min by the start of an earthward flow burst with an amplitude of $100-500 \mathrm{~km} / \mathrm{s}$ and a duration of 1-7 min. The dipolarizations are accompanied by large amplitude fluctuations in the field as reported earlier by AMPTE/CCE (Ohtani et al., 1992).

We also note the sharp $B_{z}$ increase at GOES 9 at $\sim 07: 58$ UT. This dipolarization follows by $\sim 1$ min the start of a flow burst at WIND and then, $\sim 1 \mathrm{~min}$ after the GOES 9 dipolarization, a sharp dipolarization of the magnetic field at WIND is observed. This chain of events in which the earthward flow burst is followed by a "pile-up" of dipolar flux tubes first at geosynchronous orbit and then, later, at greater distances in the tail is in good qualitative agreement with the MHD simulations of Hesse and Birn (1991). The downtail propagation speed for the magnetic field pile-up in this event is high, $\sim 850 \mathrm{~km} / \mathrm{s}$, relative to the observed flow speeds, but low with respect to the Alfven speed in the lobes.

\section{Summary}

In this study we have examined a unique set of solar wind, near-earth and ground-based observations returned by Interball, WIND, GEOTAIL, GOES 9, and CANOPUS during a substorm. Despite their large radial and azimuthal separations all three tail spacecraft observed a near-simultaneous 1-2 min southward perturbation of the magnetic field at substorm onset. The exact manner in which this perturbation is produced will have to be the 


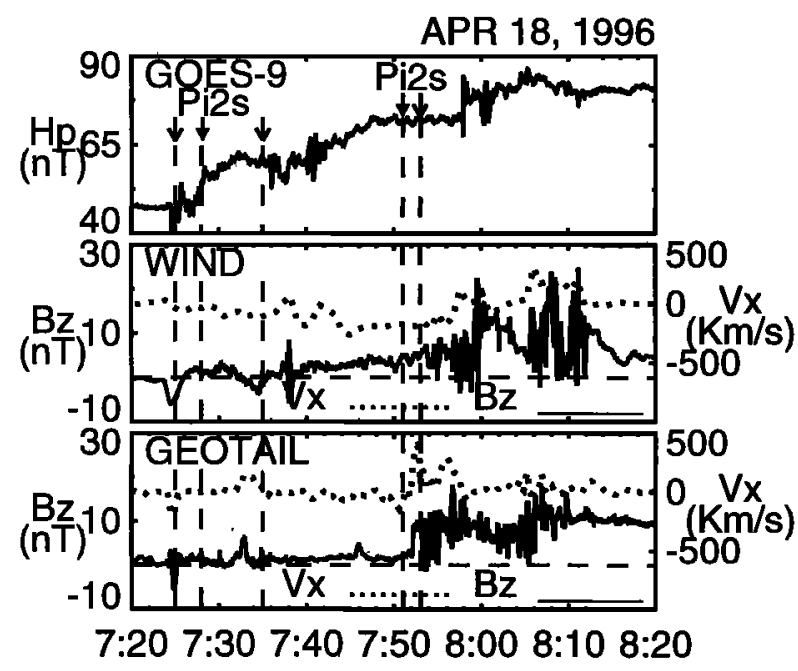

Figure 3. An expanded view of the magnetic field dipolarization within the substorm current wedge using high resolution magnetic field measurements.

subject of further analysis and modeling. However, the comparability of the perturbation amplitude at all three spacecraft suggest that the source region must have large scale lengths and/or be distant relative to the 5-10 $R_{c}$ spacing of the three spacecraft observing the southward field pulse.

The timing of the April 18th dipolarization events and BBFs relative to substorm onset is very similar to that observed in previous studies. It was not until $\sim 25-30 \mathrm{~min}$ after substorm onset that a series of very rapid dipolarizations were observed at both WIND and GEOTAIL. These dipolarization events were preceded by $\sim 1-2$ min by the start of $\sim 1.7$ min bursts of earthward flow with peak speeds of $100-500 \mathrm{~km} / \mathrm{s}$. More importantly, temporally overlapping BBFs and dipolarization events were observed at WIND and GEOTAIL near the peak of the expansion phase. While previous studies by Angelopoulos and co-workers have shown that these flow bursts must be spatially localized, our observations with two spacecraft strongly suggest that the number and/or azimuthal extent of these regions may grow during expansion phase to produce overlapping events at these two widely separated spacecraft. However, it must also be noted that WIND, which stayed in the cross-tail current sheet for about another $1 \mathrm{hr}$, observed several more BBF events, each accompanied by a dipolarization event and/or other large amplitude perturbations in the magnetic field. These latter observations suggest that in the substorm recovery phase the number and/or extent of earthward flow channels may decrease and become difficult to observe with a limited number of spacecraft.

These unique ISTP observations confirm the close relationship between bursty bulk flows and magnetic field dipolarizations during substorms first observed in AMPTE and ISEE data by Baumjohann et al. (1990) and Angelopoulos et al. (1992) and in MHD simulations by Hesse and Birn (1991). Our results add to this picture by suggesting an azimuthal spreading in the local time extent and/or number of earthward flow channels as the peak of the expansion phase is reached. In future studies of the April 18, 1996 substorms we will attempt to further examine these relationships and to conduct careful comparisons of the observations against the predictions of the various substorm models with respect to the development of the SCW and fast flows in the nearearth plasma sheet (e.g. Hesse and Birn, 1991; Lui, 1996; Rostoker, 1996).

Acknowledgements. We wish to thank all of those who have contributed to the success of the International Solar Terrestrial Physics Program which makes such research possible. The assistance of C. Liebrecht and J. Byrnes with computational aspects of this study is gratefully acknowledged. Conversations with M. Hesse and A.T.Y. Lui and suggestions by both referees also contributed to this work. The UC Berkeley WIND effort is supported in part by NASA grant NAG5-2815. The work of G.R. was supported by the Natural Sciences and Engineering Research Council of Canada. The CANOPUS instrument array was constructed by and is maintained and operated by the Canadian Space Agency.

\section{References}

Angelopoulos, V., et al., Bursty bulk flows in the inner central plasma sheet, $J$. Geophys. Res., 97, 4,027, 1992.

Angelopoulos, V., et al., Multipoint analysis of a bursty bulk flow event on April 11, 1985, J. Geophys. Res., 101, 4,967, 1996.

Baumjohann, W., G.G. Paschmann, and H. Luhr, Characteristics of high-speed ion flows in the plasma sheet, J. Geophys. Res., 95, 3,801, 1990.

Dungey, J.W., Interplanetary magnetic field and the auroral zones, Phys. Rev. Lett., 6, 47, 1961.

Fairfield, D.H., Multi-point measurements of magnetotail dynamics, Adv. Space res., 8, 97, 1988.

Hesse, M., and J. Birn, On dipolarization and its relation to the substorm current wedge, J. Geophys. Res, 96, 19,41, 1991.

Jacquey, C., et al., Location and propagation of the magnetotail current disruption during substorm expansion: Analysis and simulation of an ISEE multi-onset event, Geophys. Res. Lett., $18,389,1991$.

Kokubun, S., and R.L. McPherron, Substorm signatures at synchronous altitude, J. Geophys. Res., 86, 11,265, 1981.

Lopez, R.E., et al., Midtail plasma flows and the relationship to near-earth substorm activity: A case study, J. Geophys. Res., 99, 23,561, 1994.

Lui, A.T.Y., Current disruption in the Earth's magnetosphere: Observations and models, J. Geophys. Res., 101, 13,067, 1996.

Ohtani et al., Initial signatures of magnetic field and energetic particle fluxes at tail reconfiguration: Explosive growth phase, J. Geophys. Res., 97, 19,311, 1992.

Rostoker, G., Phenomenology and physics of magnetospheric substorms, J. Geophys. Res., 101, 1996.

J. A. Slavin, D. H. Fairfield, R. P. Lepping, A. Szabo, M. J. Reiner, and M. L. Kaiser, Laboratory for Extraterrestrial Physics, NASA/GSFC, Greenbelt, MD 20771

C. J. Owen, Astronomy Unit, Queen Mary and Westfield College, London E1, 4N3, UK

T. Phan, and R. Lin, Space Sciences Laboratory, University of California, Berkeley, CA 94720

S. Kokubun, Solar-Terrestrial Environment Laboratory, Nagoya Univ., Toyokawa 442, Japan

T. Mukai, and T. Yamamoto, Institute for Space and Astronautical Sciences, 3-1-1 Yoshinodai, Kanagawa 229, Japan

H. J. Singer, NOAA Space Environment Center, 325 Broadway, Boulder, CO 80303

S. A. Romanov, Space Research Inst., Russian Academy of Sciences, ul. Profsoyuznaya 84/32, Moscow, 117810, Russia

J. Buechner, Max-Planck-Institut fuer Aeronomie, D-37191

Katlenburg-Lindau, Germany

T. Iyemori, Kyoto University, Kyoto, Japan

G. Rostoker, Department of Physics, University of Alberta, Edmonton, AB T6G 2J1, Canada

(Received October 3, 1996; revised February 6, 1997; accepted February 8, 1997.) 